\title{
The Official Website of the AMS Experiment
}

\author{
Matthew Behlmann ${ }^{1}$, Maxim Konyushikhin ${ }^{1}$, Andrey Pashnin ${ }^{1}$, Baosong Shan ${ }^{2, *}$, Jiahui \\ $\mathrm{Wei}^{3, * *}$, Yonghuan $\mathrm{Xu}^{4}$, and Zhaoyi $\mathrm{Qu}^{5}$ \\ ${ }^{1}$ Massachusetts Institute of Technology, Laboratory for Nuclear Science, Cambridge, MA 02139, \\ United States \\ ${ }^{2}$ Beihang University, School of Mathematical Sciences, Beijing 100191, China \\ ${ }^{3}$ DPNC, Université de Genève, CH-1211 Genève 4, Switzerland \\ ${ }^{4}$ Southeast University (SEU), Nanjing, 210096, China \\ ${ }^{5}$ Institute of Physics, Academia Sinica, Nankang, Taipei 11529, Taiwan
}

\begin{abstract}
The Alpha Magnetic Spectrometer (AMS-02) is a particle physics experiment installed on board the International Space Station (ISS). It has been operating since May 2011 and is expected to continue through 2028 or beyond. The AMS collaboration seeks to store, manage and present its research results as well as details about the detector and operations. An open-source content management framework is utilized as the platform to build the website. This platform allows management of a variety of information, such as institutes in the collaboration, physics results, publications, academic events, etc. This note discusses the motivation, strategy, infrastructure, web design techniques, custom modules, data sharing plan, and day-to-day operation. The resulting website is located at https://ams02.space.
\end{abstract}

\section{Introduction}

The Alpha Magnetic Spectrometer (AMS-02) is a general-purpose high-energy particle physics detector. It was installed on the International Space Station (ISS) on 19 May 2011 to conduct a unique, long-duration mission of fundamental physics research in space [1]. An official website is needed to provide up-to-date information on AMS.

In this stage of development, the website targets the physics community, providing essential knowledge of the AMS detector and physics, as well as an archive of publications and published data. News and events concerning AMS are also included and kept updated [2].

The website is hosted at CERN in Geneva, Switzerland, where the AMS Payload Operation Control Center (POCC) is located. CERN Web Services provides centralized web servers and web authoring technology to simplify development. Drupal 8 is the content management system (CMS) for CERN websites, and it enabled the website team to build a user-friendly and easy-to-maintain official site.

\footnotetext{
*e-mail: baosong.shan@cern.ch

**e-mail: jiahui.wei@cern.ch
} 


\section{Content management system and Drupal}

A CMS is a software tool that lets users add, publish, edit, or remove content from a website via web browser. Typically, the CMS is written in a scripting language and runs on a machine containing a database and a web server. Website content and settings are stored in database, and for each page request that comes to the web server, the scripts combine information from the database and assets (JavaScript files, CSS files, image files, etc.) to build the page. The assets are either part of the CMS or have been uploaded separately.

Drupal is a flexible CMS based on the LAMP stack (Linux, Apache, MySQL, PHP/Perl/Python), with a modular design allowing features to be added and removed by installing and uninstalling modules. A module consists a set of PHP, JavaScript, and/or CSS files [3]. The platform also allows to modify the look and feel consistently across all pages. As one of the most popular CMS's on the market, Drupal has an active community with more than 44000 contributed modules.

\section{Website development}

\subsection{Workflow}

Development of the AMS website was scheduled to take three months, beginning in March 2019. The team reserved a one-month review period before the launch day in July 2019 since the contents needed to be reviewed by the collaboration. Therefore the website was expected to be functional before the review and an extensive amount of content needed to be processed.

As shown in Fig. 1, the website development workflow was divided into three disciplines: developers, themers, and site builders.

- Developer: a developer is responsible for the functionality of the site, including management of public modules and the development of customized modules.

- Themer: a themer is responsible for the look and feel of the site, including layout, typography, interactive design, etc.

- Site builder: a site builder is responsible for the structure and the contents of the site, including organization of the raw materials, navigation and daily maintenance.

The flexibility of Drupal enables the website development team to carry out site building and content processing in parallel. A basic functional development site is prepared quickly for site builders to start editing the contents. The developers and themers work on several testbed sites in collaboration with site builders to improve the site and to meet new requirements. As the website is proved to be robust at the final stage of development, editing work is gradually switched to the production site. This guarantees the future operations will not be affected by new feature development.

\subsection{Website structure and navigation}

To organize and display a variety of contents, a three-level structure is used, as presented in Table 1. The topics are classified into three categories, which make up the level-1 structure of the website and are displayed on the top menu bar to help users find the corresponding page quickly.

The main navigation of this structure is facilitated by the Mega Menu module [4], a tool developed by the CERN web team, which allows management of complicated structures while remaining flexible. An example of the Mega Menu is shown in Figure 2. 


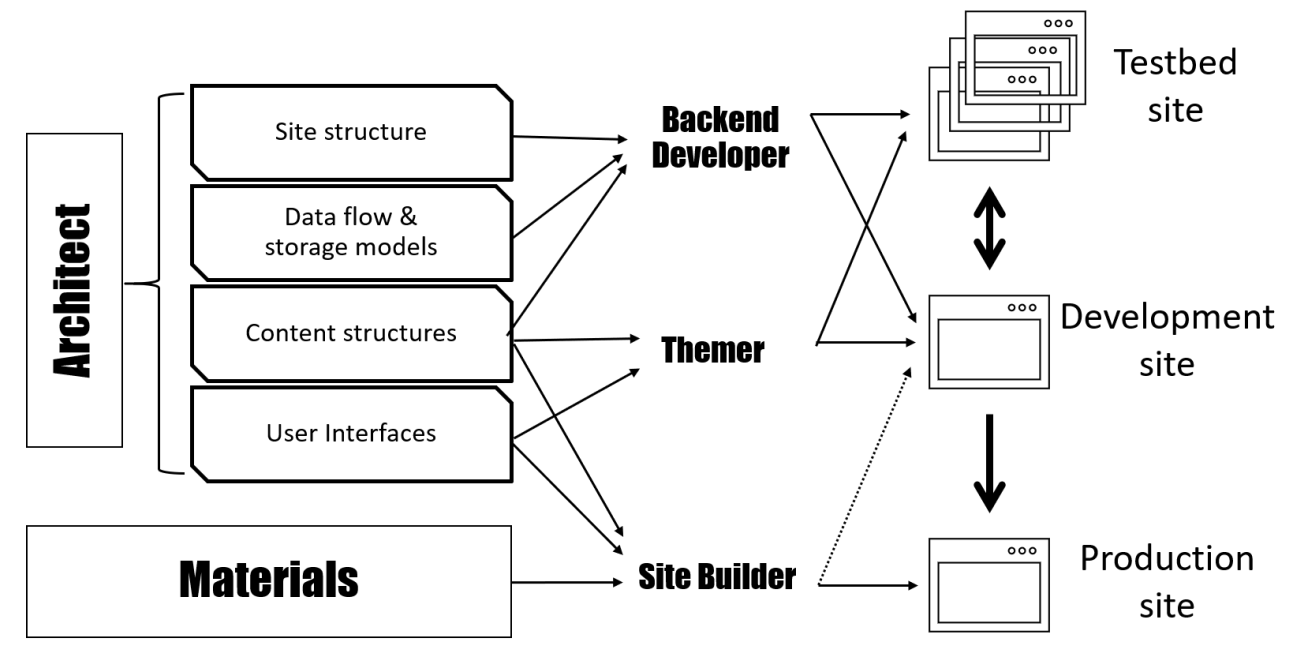

Figure 1. Website development workflow. Assignments are linked to three distinct roles. During the development, the production site is protected using a development site and several testbed sites. Sites can be synchronized once a major development update is accomplished.

Table 1. Structure of the AMS website.

\begin{tabular}{|l|l|l|}
\hline Level-1 & Level-2 & Level-3 \\
\hline Discover & What's AMS & AMS in a nutshell, AMS-01, etc. \\
& $\begin{array}{l}\text { Collaboration } \\
\text { Detector }\end{array}$ & $\begin{array}{l}\text { Institutes and collaborators. } \\
\text { Sub-detectors and operation. }\end{array}$ \\
\hline Research & $\begin{array}{l}\text { Physics } \\
\text { Publications } \\
\text { Advances in data analysis }\end{array}$ & $\begin{array}{l}\text { Physics topics covered by AMS research. } \\
\text { List of AMS Publications. } \\
\text { Improvements in the data analysis. }\end{array}$ \\
\hline News \& Events & $\begin{array}{l}\text { AMS days } \\
\text { AMS sessions in conferences }\end{array}$ & $\begin{array}{l}\text { Event details. } \\
\text { Programs and other details. } \\
\text { Press releases, news, etc. }\end{array}$ \\
& News & \\
\hline
\end{tabular}




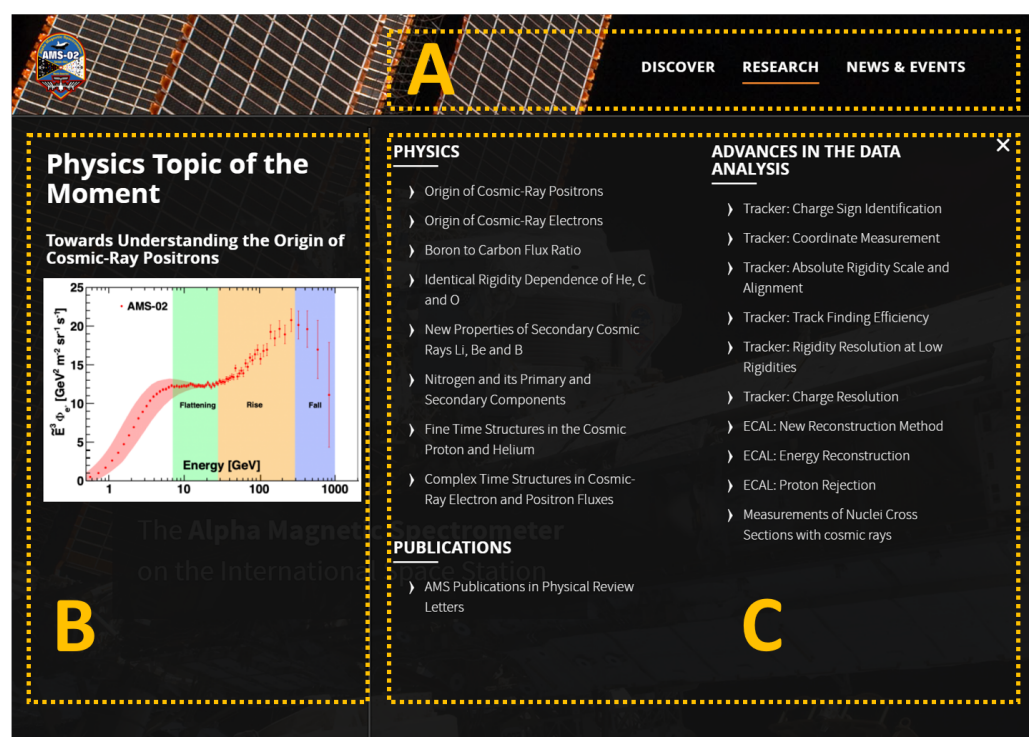

Figure 2. Layout of the Mega Menu. The menu is divided into three parts: (a) the top menu bar fixed to the top of the page. The menu panel is expanded by clicking on the menu bar. (b) the highlight area to promote featured contents and (c) the main menu area with all the items included under the selected category.

\subsection{Modular content}

Since the Drupal database stores contents modularly, site builders can insert and modify fragments instead of editing a whole page of formatted text. This will simplify future updates and maintenance but also requires a plan for the content structures.

Ideally, though the contents can be displayed in any of various complex formats, the same fundamental blocks of information point to a single place. An example is the institute and collaborator information. In a piece of institute content, it stores the address as plain text, the location as longitude and latitude data, the introduction paragraph as formatted text, etc. Similarly, collaborator contents store name, email, etc., as separate fields. For each collaborator, a special reference field is used to link the person to the institutes in the database with which they are affiliated. Therefore the institute information is displayed in a collaborator's personal page accordingly.

The modular contents also allow creating collective displays. In Drupal this function is supported by a core module called View, which allows administrators and site designers to create, manage, and display lists of contents. In one particular case, we wished to display all collaborators associated with an institute as a list after the its information. This is implemented by the View module using the reference information stored for each collaborator. The fields to be displayed and the wrapping styles can also be customized within the module. The View module also has a filtering and sorting function to define the sequence and grouping of the collaborator names.

\subsection{Theme development}

The AMS website look is based on a customized theme developed by the CERN web group. Two snapshots of the theme are shown in Fig. 3. 


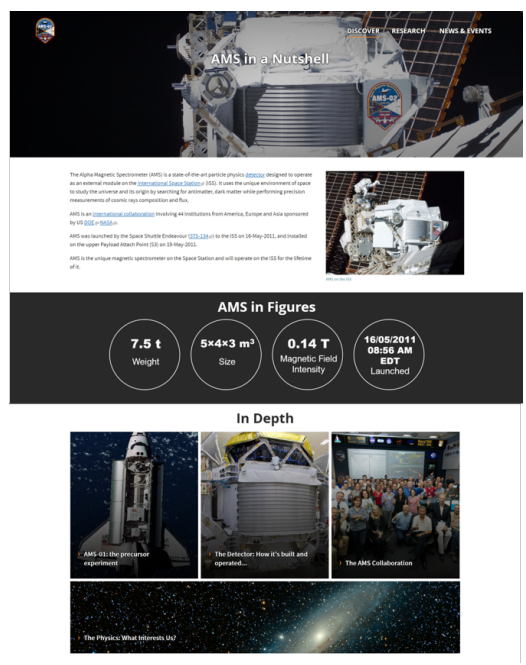

(a)

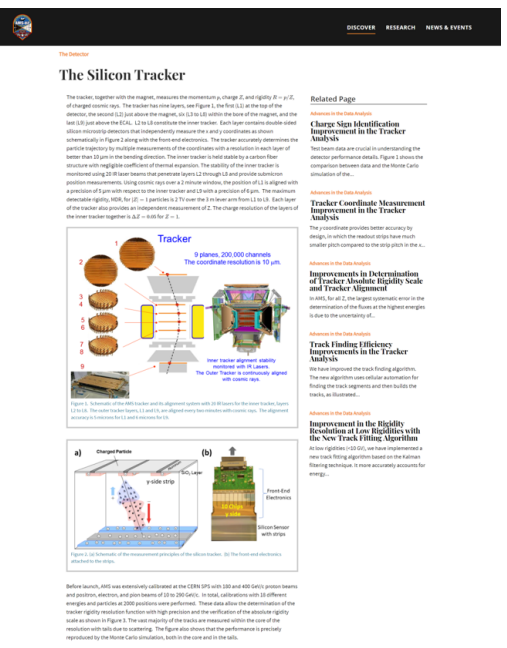

(b)

Figure 3. Two snapshots of the AMS theme. Page (a) is an introduction page with dedicated design using the Paragraph module. This module enables site builders to use predefined Paragraph Types to order contents in a page. Page (b) is generated by the Display Suite module where a common template is applied to all similar pages.

Responsive web design is also used to make web pages render well on a variety of devices and window or screen sizes. The theme is built on the Bootstrap framework [5], an opensource development toolkit with HTML, CSS, and JavaScript. Bootstrap provides a grid system using containers, rows, and columns to lay out and align contents. The predefined style ensures that all visual elements are fully responsive. Figure 4 shows a comparison of the publication list displayed on different devices.
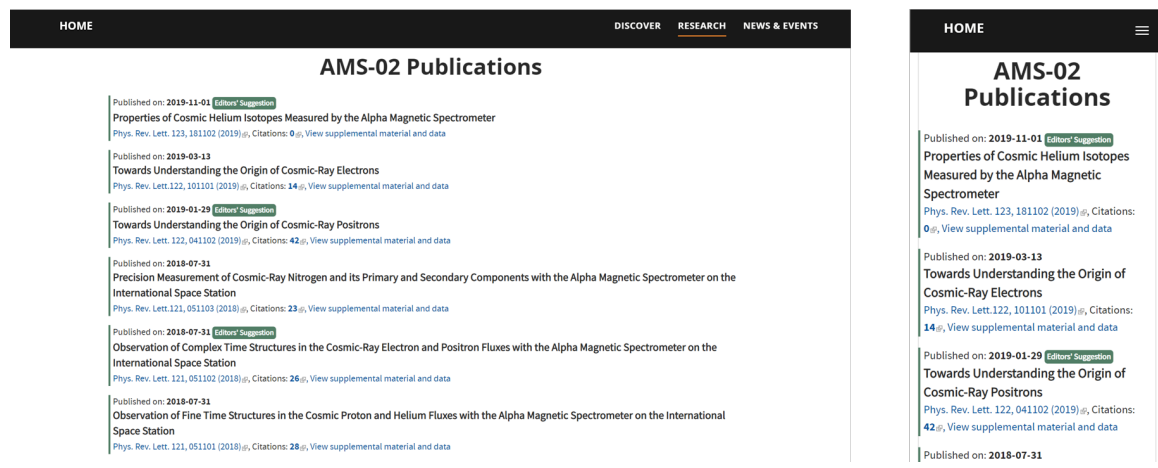

Figure 4. A comparison of the list of AMS publications displayed on a desktop computer and on a mobile phone. The Bootstrap grid system is used instead of the traditional HTML tables to implement responsive design. Visual element rendering is based on the screen width. 


\subsection{Customized module development}

Several self-developed modules are also used to add functionality. The Event Count module reads the tally of physics events collected by AMS from the production server and is updated every 20 minutes. The Citation Number module uses the API of INSPIRE [6], a hub for scholarly information in the high-energy physics community, to retrieve and display the current citation count of each AMS publication. There are also modules providing new data formats designed for the AMS contents.

\subsection{Machine-readable data distribution}

To provide machine-readable data to the physics community, the AMS website distributes comma-separated values (CSV) and plotting macros to reproduce the figures and tables in the supplemental materials of AMS publications.

Furthermore, the AMS website provides links to two external databases containing cosmic ray data from a large number of experiments [7,8]. The two databases include the AMS published fluxes of electrons, positrons, and nuclei as a function of energy, rigidity, or kinetic energy per nucleon. Users can visualize AMS data and compare with other experiments through the web interface. Dedicated links to these datasets can be found in the AMS website under the corresponding publication.

\section{Launching and Operation}

The official website of AMS was opened to public in July 2019 after 105 days of development. Since then, the team began to focus on the daily operation of the website.

Search engine optimization (SEO) has remained an essential task throughout the development process. The new website was submitted to Google, Bing, Baidu and Yandex for indexing on the launch day. Direct links from NASA, CERN, Wikipedia and the collaborating institutes were also added. The AMS website began to appear on the first page of mainstream search engines within one month after launch.

The website is monitored with the Piwik Web analytics module, an open-source web application. The Piwik dashboard is integrated in the CERN web management toolkit. The referrer statistics, as presented in Fig. 5, show that a majority of users accessed through direct link during the first month after launch. The share of visitors from search engines increased with SEO efforts, and this is now the second largest source of visitors.

An increasing number of visitors are from outside the physics community. A website promotion banner is installed at the POCC at CERN, which also serves as a visit point open to the public. The website provides supplementary information to visitors. The AMS website is also referenced by scientific news providers to provide in-depth information.

\section{Conclusion}

The AMS official website built with Drupal provides essential information on AMS, including 35 articles introducing the detector and research works of AMS, the information of 44 institutes and more than 250 collaborators, the collection of AMS publications and up-to-date news and events related to AMS. To date, the website has reached an average of more than 40 daily visits. The website keeps evolving and will extend its target audiences in future versions. 


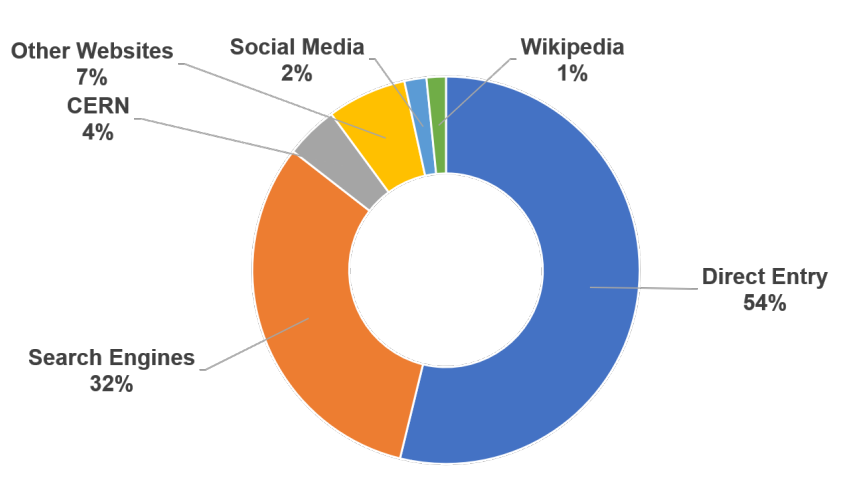

Figure 5. The referrer statistics from Piwik. The majority of the website visitors is through direct entry and the secondary source is search engine.

\section{References}

[1] A. Kounine, Int. J. Mod. Phys. E 21, 1230005 (2012)

[2] AMS-02 Official Site [https://ams02.space] (2019)

[3] Drupal 8 User Guide [https://www.drupal.org/docs/user_guide/en/index.html] (2019)

[4] Mega Menu [https://drupal-tools.web.cern.ch/docs/modules/mega-menu] (2019)

[5] Bootstrap Website [https://getbootstrap.com/] (2019)

[6] INSPIRE Website [http://inspirehep.net/] (2019)

[7] V. Di Felice, C. Pizzolotto et al., PoS (ICRC2017), 1073 (2017)

[8] D. Maurin, F. Melot, R. Taillet, A\&A 569, A32 (2014) 